\title{
Desarrollo y futuro del Programa Ampliado de Inmunizaciones en Chile
}

\author{
DEVELOPMENT AND FUTURE OF THE EXPANDED PROGRAMME ON \\ IMMUNIZATION IN CHILE
}

M. TERESA VALENZUELA B. ${ }^{1}$

\section{INTRODUCCIÓN}

Al reflexionar sobre esta pregunta puedo disfrutar acerca de un pasado, de un presente y proyectarme a un futuro. Un futuro....., por supuesto, pues este programa se basa en principios indelegables e impostergables de la sociedad dado que constituye un derecho de todos los niños, ha demostrado logros indiscutibles tales como la disminución de la morbilidad y mortalidad de las enfermedades inmunoprevenibles contribuyendo a la disminución de la mortalidad infantil, ha cruzado los distintos niveles de la población independientemente de su condición social, de accesibilidad a los sistemas de salud, pobres, de áreas rurales, estando por sobre las iniquidades sociales; en fin, ha demostrado en el tiempo ser una de las herramientas de salud pública de mayor beneficio en la ecuación costo-efectividad.

Describimos a continuación líneas posibles de desarrollo de un programa consolidado, que ha demostrado sus logros y las que en último término están sujetas al análisis y discusión de un equipo de trabajo en la instancia ministerial y a la aprobación y autorización por parte de las autoridades de salud. Sin embargo, para plantear estas líneas es necesario describir la situación actual alcanzada mediante este programa reconocido como de Bien Público Nacional.

\section{ANTECEDENTES DEL PROGRAMA}

Para llevar a cabo en forma programática las acciones de inmunización en Chile, las cuales ya habían constituido historia en nuestro medio, se estableció una programa a partir de 1978, cuatro años después de la aprobación de la creación de los Programas Ampliados de Inmunizaciones en todos los países del mundo (Asamblea WHA 2757), en mayo de 1974.

En su inicio, este programa incorporó las vacunas contra las seis principales enfermedades consideradas asesinas para la población infantil: difteria, coqueluche, tétanos, poliomielitis, tuberculosis y sarampión. Se establecieron las políticas acerca de la obligatoriedad, gratuidad, garantía por parte del Estado de ser un derecho de la población y un deber de éste, y el financiamiento para llevarlo a cabo.

A lo largo de la historia, este programa se sustenta en la organización de salud con una amplia base operativa, especialmente a partir de 1952, gracias a la creación del Servicio Nacional de Salud.

Sin embargo, cabe destacar que en la primera mitad del siglo pasado Chile había introducido la vacunación anti variólica, piedra angular en la eliminación de la enfermedad en 1950, 17 años antes que ella fuera declarada como erradicada del mundo, y que en 1949 incorporó la vacuna anti tuberculosa, BCG.

Actualmente proporciona en forma gratuita las va-

\footnotetext{
${ }^{1}$ División de Epidemiología, Escuela de Salud Pública. Unidad de Investigación Clínica Integrada y Etica de la Investigación, Facultad de Medicina, Universidad de Chile. Centro de Vacunas en Desarrollo de Chile (CVD-Chile).
} 
cunas objeto del PAI, las que ascienden a nueve antígenos, para toda la población infantil que habita en Chile y que tiene menos de 7 años de edad, a través de los establecimientos de atención primaria de salud. Estos antígenos son los seis ya señalados a los que se han adicionado rubéola, parotiditis y Haemophilus influenzae $\mathrm{b}$. Adicionalmente, en forma de campañas de vacunación, se otorga la vacuna contra influenza dirigida a grupos de riesgo, y desde 1990 se inició un programa de vacunación contra la hepatitis B. Esta última ha sido indicada para el personal de salud en riesgo de adquirir la infección, cubriendo desde ese año en adelante distintos grupos de acuerdo al tipo de actividad que realizan.

Chile es uno de los países que ha acumulado una larga y exitosa trayectoria en materia de inmunizaciones logrando a través de ellas la erradicación de la viruela, como ya se señaló, en el año 1950. Posteriormente se logró la erradicación de la poliomielitis en 1975.

Más adelante, en el plan de acción de este programa se ubica la eliminación del sarampión, poniendo en operación este plan a contar de 1992.

\section{Estrategias del PAI en Chile}

Las estrategias utilizadas para llevar a cabo este programa han sido entre algunas,

- utilizar la organización y estructura del Sistema de Salud Pública de Chile;

- contar con el respaldo de las acciones a través de Decretos Supremos firmados por el Presidente de la República, es decir contar con el respaldo político independientemente de los gobiernos existentes;

- desarrollo de las actividades del PAI integradas al Programa Infantil, lo que ha permitido hacer más eficientes los recursos disponibles;

- la adquisición centralizada de las necesidades de vacunas para el país y su disponibilidad permanente;

- la capacitación, la supervisión y la evaluación anual desarrolladas en los tres niveles: local, intermedio y central;

- la mantención de un catastro de elementos de cadena de frío y monitoreo de coberturas de vacunación en zonas de riesgo;

- la coordinación intersectorial con diferentes entidades;

- la creación de una Comisión Nacional Asesora del PAI, cuyos integrantes representan a las Universidades, a Servicios de Salud, Sociedades Científicas y al Instituto de Salud Pública, y, el compromiso del sector privado e instituciones tales como hospitales de las Fuerzas Armadas, Servicios Delegados y Universidades;

- la existencia de un programa de vacunación calendarizado, universal y gratuito para toda la población infantil el que se detalla a continuación en la Tabla 1.

Las metas propuestas por este programa en estos últimos años se han centrado fundamentalmente en:

- Disminuir la morbilidad y la mortalidad de las enfermedades prevenibles por vacunas que estén incorporadas en el programa.

- Mejorar las coberturas de vacunación con vacuna DPT - polio y trivírica mediante la vacunación del $95 \%$ de los niños bajo 18 meses de edad, con el fin de certificar la erradicación de poliomielitis, sarampión y tétanos neonatal.

- Eliminar la tuberculosis meníngea en el menor de 4 años, difteria y tétanos.

- Incorporar la vacuna contra H. influenzae b (Hib), situación consolidada el $1^{\circ}$ de Julio de 1996. El objetivo planteado fue erradicar las enfermedades invasoras causadas por este patógeno.

- Controlar la coqueluche.

- Eliminar el síndrome de rubéola congénita.

- Aumentar las coberturas de vacunación contra influenza y mejorar la orientación acerca de la indicación de ella hacia los grupos más vulnerables de la población: enfermos crónicos, adulto mayor y personal de salud.

- Mantener niveles de protección adecuada de la población mediante refuerzos de vacunación. Esta meta se ha operacionalizado, a modo de ejemplo, mediante la ejecución de campañas de vacunación masiva contra el sarampión con el objetivo de erradicar la enfermedad. Estas campañas masivas deberán continuarse con campañas de seguimiento focalizadas a los grupos más vulnerables donde se acumulan los susceptibles.

\section{Tendencia de las enfermedades inmunoprevenibles}

El éxito alcanzado por este programa se refleja claramente en la reducción de la morbilidad y mortalidad ocasionada por las enfermedades inmunoprevenibles. Sarampión. Cuarenta años atrás el sarampión era uno de los problemas más graves que existía en la pediatría y causaba los mayores estragos en la población infantil. Antes de la década de 1960, la morbilidad y 
Difteria. En estos últimos 50 años el descenso ha sido de 2.966 casos anuales con una tasa de $39 \mathrm{x}$ 100.000 habitantes a 2 casos diagnosticados en 1995 con una tasa de $0,02 \times 100.000$ habitantes. La vacuna se introdujo en 1954; sin embargo, el descenso en la morbimortalidad se produjo 7 años más tarde, en 1961. El último fallecido fue en 1991. La eliminación de la difteria es una de las próximas metas del PAI. Por esta razón es necesario enfatizar el incremento de las coberturas de vacunación por sobre $95 \%$ en cada una de las comunas del país.

Tétanos. Los casos se notifican desde 1960. El mayor número de casos notificados fue en 1963 con 73 casos. El descenso ha sido sostenido de 1982 a la fecha, desde 36 casos a 10 notificados en 1995, esta última cifra corresponde a una tasa de $0,1 \times 100.000$ habitantes, cifra que se mantiene hasta 1999. De 10 casos notificados en 1994, uno correspondió a tétanos neonatal. En 1995, por primera vez no se notificó ningún caso de tétanos neonatal en Chile. El toxoide tetánico se introdujo en forma programática a contar de 1975, asociado al toxoide diftérico (DPT).

Después de esta breve reseña de la tendencia de las enfermedades inmunoprevenibles en Chile, queda claro el sustento del éxito de este programa.

\section{Proyecciones y desafíos del PAI en Chile}

Sin embargo, la gran pregunta es: ¿podemos hacer algo más?. La respuesta que daría a ésta, es por supuesto que sí. ¿En qué áreas? En las coberturas de vacunación, en los sistemas de registro e información, en la vigilancia epidemiológica tanto orientada a la enfermedad como a la seguridad de las vacunas, en la gestión del programa, en ampliar el concepto y beneficio de la protección a otros grupos de la población, en la introducción de nuevas vacunas o combinaciones de ellas y en la investigación.

Cobertura de vacunación. Este concepto debe transformarse en el eje central del diseño y evaluación del programa de inmunizaciones. Mejorar las coberturas implica tener un conocimiento acabado de la población menor de 7 años de edad bajo la responsabilidad de cada comuna, estrategias para alcanzar a todos ellos, fortalecer los sistemas de comunicación a la población en forma permanente acerca de la importancia y beneficio de las vacunas para la salud de la población. Para ello se debiera monitorear periódicamente las poblaciones por grupos de edad y por comuna, de modo de tener el denominador más cercano a la realidad.

Una forma de obtener ese dato es a través del co- nocimiento de los recién nacidos vivos (RNV) por comuna y aplicar a ellos la fórmula de ajuste (E. Taucher).

Actualmente, según datos oficiales de coberturas de vacunación de los años 1997, 1998 y 1999 se podría estimar que en el trienio: $73.756 \mathrm{RNV}$ no recibieron la BCG, con un promedio anual de $24.585 \mathrm{RNV}$ por año, 82.440 no recibieron la tercera dosis de DPTpolio, $H i b$, con un promedio anual de 27.480 niños menores de un año y 91.349 no recibieron la primera dosis de la vacuna trivírica, con un promedio anual de 30.450 niños de un año de edad. A estos crudos números deberían adicionarse los niños no inmunizados, es decir que habiendo recibido la vacuna no respondieron efectivamente a ella; de esta forma tendríamos el total de susceptibles. Por esta razón la primera prioridad la constituye el conocimiento del denominador real y mejorar lo que hoy se dispone en materia de programa. Para mejorar la adherencia de los padres al programa es necesario efectuar un refuerzo positivo continuo a la población, con contenidos acerca de los logros de las inmunizaciones en el tiempo. Un sistema fácil y económico de comunicación sería disponer de videos educativos en todos los consultorios del país en las salas de espera. En la Región Metropolitana, al menos, casi la totalidad de los consultorios cuentan con equipos de videos. Otra estrategia para mejorar las coberturas sería el disponer de vacunas combinadas que permitan reducir el número de visitas y el número de punciones a aplicar a los lactantes.

Sistemas de registros. Teniendo en claro el método más exacto para determinar la población, el segundo paso sería implementar un sistema computacional de registro de dosis de vacunas recibidas por niño. El ideal sería registrar el Rol Unico Nacional (RUN) del niño con las dosis puestas, fecha de vacunación, los lotes de las vacunas y la comuna de residencia del niño. Este registro informatizado podría ser derivado diariamente vía electrónica a los municipios y al Servicio de Salud correspondiente. Si existen niños no vacunados habría que buscar las estrategias de acceder a ellos.

Vigilancia epidemiológica. Lo más importante y un desafío permanente, es el fortalecimiento de ella. La vigilancia epidemiológica juega un rol fundamental en el conocimiento de la carga de enfermedad de un problema determinado y en el impacto y efectividad que tiene el programa sobre ella. Además la colección sistemática de la carga de enfermedad orienta hacia el desarrollo de vacunas necesarias en el futuro, con una visión de salud pública. En este 
fortalecimiento se debe considerar la permanente supervisión y capacitación de las unidades notificadoras, el funcionamiento permanente e interacción de los equipos de la vigilancia en el nivel local y en los Servicios de Salud: epidemiólogo, pediatra, infectólogo, microbiólogo, estadístico, equipo de infecciones intrahospitalarias; un conocimiento acabado del nivel donde se producen las consultas y se resuelve mayoritariamente el problema bajo vigilancia y el método de diagnóstico de mayor sensibilidad y especificidad. Además esta vigilancia se vería enriquecida mediante la realización de estudios seroepidemiológicos. Existen métodos que permiten verificar la seroconversión o respuesta protectora a las vacunas. Actualmente se han desarrollado métodos no invasores, a través de muestras de saliva, que permiten verificar la inmunoconversión que sigue a la vacunación. Disponer de estos métodos, permitiría evidenciar la prevalencia de individuos de edades seleccionadas que poseen anticuerpos protectores específicos frente a rubéola y a sarampión, a modo de ejemplo.

Otra área a considerar en la vigilancia epidemiológica es la vigilancia de los eventos adversos a las vacunas, con una definición clara de qué se va a vigilar, cómo, quien va a analizar la información, en qué niveles y cómo se va a retroalimentar el sistema. Esto permitirá tener seguridad del producto, que sobretodo, en materia de inmunizaciones es de aplicación masiva, es decir, simultáneamente se está aplicando el mismo lote en distintos puntos del país. Gestión. Un aspecto relevante lo constituye la seguridad del presupuesto nacional para la adquisición de las vacunas; la permanencia, estímulo y perfeccionamiento del recurso humano; el sustento logístico del programa y cumplimiento de actividades, tales como, la supervisión y la capacitación. Avances tecnológicos en la preparación de vacunas, tales como su mejor estabilidad, hacen necesario una exhaustiva revisión y actualización de las normas de cadena de frío evitando pérdidas innecesarias de vacunas. Actualmente se dispone de vacunas polio con etiquetas sensibles a la temperatura llamados monitores de viales de vacuna, los que se modifican de color cuando la vacuna ha sido expuesta al calor. Es necesario además, contar con una normativa para el desecho del material cortopunzante utilizado en los procedimientos. En materia de gestión hoy en día lo más adecuado es evaluarla permanentemente en términos de indicadores seleccionados y métodos definidos de monitoreo en coberturas de vacunación, oportunidades perdidas, calidad de la cadena de frío, metas de reducción de la morbilidad y mortalidad de las enfermedades inmunoprevenibles, costos por niño completamente inmunizado, entre algunos.

Participación. Otro aspecto relevante en el desarrollo de este programa, caracterizado por su dinamismo y flexibilidad, es considerar la participación del médico en el equipo del PAI de los Servicios de Salud. Es importante recordar que en nuestra cultura, como en muchos otros países, la prescripción médica es de un alto grado de cumplimiento por parte de la población. Además debido al crecimiento importante del subsistema privado cuya cobertura asciende a $27 \%$, se torna imprescindible la buena coordinación, integración, supervisión y capacitación de este sector. Incorporación de nuevas vacunas. Es deseable que se defina una agenda de incorporación de nuevas vacunas a mediano y largo plazo, de acuerdo a prioridades de salud pública. Un área importante en la agenda de los próximos años es vacunar contra infecciones respiratorias agudas: vacunas contra Streptococcus pneumoniae, contra virus respiratorio sincicial, vacunas mejoradas contra influenza, contra agentes bacterianos que afectan el SNC, especialmente Neisseria meningitidis y sus diversos serogrupos. Otras vacunas que debieran estar presente en los próximos años son vacunas contra enfermedades entéricas como rotavirus y virus de hepatitis A. Este último grupo de vacunas tiene connotaciones de salud como desde el punto de vista de desarrollo económico visualizándonos en el contexto de país exportador de alimentos.

Es deseable que Chile se una a los esfuerzos de las organizaciones internacionales y del resto de otros países en incorporar en sus esquemas de vacunación la vacuna contra hepatitis $B$, dado que la vacunación sólo de grupos de riesgo no ha demostrado ningún éxito.

Ampliación del concepto de protección. Es necesario incorporar como objetivo global del programa el concepto de protección específica, más allá de las vacunas de la infancia. Ya es el momento de integrar en este concepto al adolescente, al adulto, a grupos de riesgo, sobre todo a los inmunosuprimidos, al personal de salud y de educación.

Investigación. Es necesario diferenciar dos tipos de investigaciones, una de carácter operativo y otra de carácter clínico en el desarrollo de nuevas vacunas. Ambos tipos debieran recibir el apoyo del sistema y de las iniciativas presentadas tanto por grupos universitarios u otro tipo de organizaciones dedicadas al tema, cautelando el buen cumplimiento de las bue- 
nas prácticas clínicas como los aspectos éticos. La investigación es la mejor aliada del progreso científico.

Adaptabilidad. Por último, un gran desafío que debe sustentar el PAI y de gran relevancia, es su adaptación a las reformas y cambios del sector salud, sin perder su efectividad y su presencia en los distintos niveles de atención.

En resumen, este es un programa que ha dejado grandes lecciones de Salud Pública en materia de prevención de enfermedades y ha engrandecido las acciones de salud en la atención primaria, por lo tanto la efectividad de éste no está en discusión. El camino a seguir es adaptarse a las modificaciones del sistema de salud del país y los matices que apuntan a una mayor eficiencia de los recursos disponibles para avanzar en la cobertura de nuevos grupos que se verían favorecidos bajo el alero del programa e incorporar nuevas vacunas cuya necesidad emerja desde la perspectiva de Salud Pública.

Las inmunizaciones en la prevención de enfermedades transmisibles de los niños han demostrado ser una herramienta en Salud Pública de la mayor costo-efectividad, revelado por el control, eliminación y erradicación de enfermedades.

\section{BIBLIOGRAFÍA}

1.- Ministerio de Salud de Chile, Anuarios de Enfermedades de Notificación Obligatoria. 1950-1998.

2.- Ministerio de Salud de Chile, Circular $N^{\circ} 4$ F/2, 25 Enero 1996. Vigilancia epidemiológica de cuadros invasores producidos por Haemophilus influenzae $b$.

3.- Ministerio de Salud de Chile, Circular No 4 F/3, 25 Enero 1996. Vigilancia epidemiológica de coqueluche.

4.- Ministerio de Salud de Chile, Circular No 4 F/14, 6 Marzo 1996. Actualización de vigilancia epidemiológica del tétanos.

5.- Ministerio de Salud de Chile, Circular $N^{\circ} 4$ F/02, 15 Enero 1997. Actualización de la vigilancia epidemiológica de las parálisis fláccidas agudas.

6.- JIMÉNEZ J. Control del sarampión en Chile. Campaña nacional de inmunización 1992. Editorial Dolmen, estudio 1995

7.- VALENZUELA M T, LEAL I, TORO J. Capítulo VII, Vigilancia epidemiológica del sarampión en Chile, en Control del sarampión en Chile, Jiménez J (editor), Edit Dolmen Estudio 1995.

8.- VALENZUELA M T, LEAL I, YOUNG V, VICENTE M, TORO J. Chile hacia la eliminación del sarampión 1992-1996. Rev Chil Infect 1997; 14: 97-108.

9.- VALENZUELA M T. Infección por Bordetella pertussis en Chile y el rol potencial de nuevas vacunas. Rev Chil Infect 1996; 13: 242-56.

10.- VALENZUELA M T, VEGA J, LEAL I, VICENTE M, YOUNG V, RAMÍREZ E. Estado inmunitario de 4 grupos de edad de población chilena contra sarampión y rubéola. Rev Méd Chile 1999; 127: 359-65.

11.- COFRE J. Bordetella pertussis: Fastidio para clínicos y epidemiólogos Rev Chil Infect 1999; 16: 7-16.

12.- LAGOS R, HORWITZ I, TORO J, et al. Large scale post licensure, selective vaccination of chilean infants with PRP-T conjugate vaccine: practicality and effectiveness in preventing invasive Haemophilus influenzae type $\mathrm{b}$ in infections. Pediatr Infect Dis $\mathbf{J}$ 1996; 15: 216-22.

13.- FERRECCIO C, ORTIZ E, ASTROZA L, RIVERA C, CLEMENS J, LEVINE M. A population-based retrospective assessment of the disease burden resulting from invasive Haemophilus influenzae in infants and young children in Santiago, Chile. Pediatr Infect Dis J 1990; 9: 488-94.

14.- ROMERO M I, VALENZUELA M T, QUIROZ O. Evaluación del Programa Ampliado de Inmunizaciones de Chile, Ministerio de Hacienda, Dirección General de Presupuestos, Agosto, 1999.

15.- JIMÉNEZ J. Vaccines-a wonderful tool for equity in health. Vaccine 2001; 19: en prensa.

16.- BROWN D W, RAMSAY M E, RICHARDS A F, MILLER E. Salivary diagnosis of measles: a study of notified cases in the United Kingdom, 1991-3. Br Med J 1994; 308: 1015-7.

17.- GEORGE J R, FITCHEN J H. Future applications of oral fluid specimen technology. Am J Med 1997; 102: 21-5.

Correspondencia a:

María Teresa Valenzuela Bravo.

E-mail: mvalenzuela@machi.med.uchile.cl 